\title{
Resolution of an Infection with Leishmania braziliensis Confers Complete Protection to a Subsequent Challenge with Leishmania major in BALB/c Mice
}

\author{
Hermenio C Lima/*, Gregory K DeKrey, Richard G Titus/+ \\ Department of Pathology, College of Veterinary Medicine and Biomedical Sciences, Colorado State University, \\ Fort Collins, CO 80523-1671, USA
}

\begin{abstract}
Both Leishmania major and L. braziliensis induce cutaneous leishmaniasis in BALB/c mice. Whereas $B A L B / c$ mice die of infection with $\mathrm{L}$. major, they cure an infection with $\mathrm{L}$. braziliensis. We report here that after curing an infection with $\mathrm{L}$. braziliensis, $B A L B / c$ mice are resistant to challenge with $\mathrm{L}$. major. When challenged with $\mathrm{L}$. major, L. braziliensis pre-treated BALB/c mice mounted a delayed-type hypersensitivity response to L. major and produced high amounts of interferon-g (IFN-g) but low amounts of interleukin-4. The IFN-gproduced by the $\mathrm{L}$. braziliensis pre-infected mice was involved in the protection seen against L. major challenge since treating the mice with a neutralizing anti-IFN-gabrogated the protection. This suggests that cross-reactive antigen epitopes exist between $\mathrm{L}$. braziliensis and $\mathrm{L}$. major and that pre-infection with $\mathrm{L}$. braziliensis primes $B A L B / c$ mice to epitopes on $\mathrm{L}$. major that can elicit a protective Thl response to the parasite.
\end{abstract}

Key words: Leishmania braziliensis - Leishmania major - mice - cross-protection - cytokines

Organisms of the genus Leishmania induce a spectrum of diseases in humans and in experimental animals. Infection of mice with L. major, one cause of cutaneous leishmaniasis, is perhaps the best studied model for cutaneous leishmaniasis (reviewed in Bogdan et al. 1993, Liew \& O'Donnell 1993, Reed \& Scott 1993, Titus et al. 1994, Reiner \& Locksley 1995). Most mouse strains cure an infection with $L$. major, however BALB/c mice are a notable exception since they ultimately die of infection with $L$. major when the disease becomes systemic. Considerable work in this model has revealed that mice that are resistant to infection with L. major develop a Th1 immune response and its associated cytokine profile [interferon-gamma $\left(\right.$ IFN-g) ${ }^{\text {hi }}$; interleukin-4 (IL-4) $\left.{ }^{\text {lo }}\right]$. IFN-gactivates L. major infected macrophages (MØs) to kill the parasite (Murray et al. 1983, Titus et al. 1984, Nacy et al. 1985). In contrast, susceptible BALB/c mice develop a Th2 response and its associated cytokine

\footnotetext{
This work was supported by the National Institutes of Health grant AI 29955.

* Present address: Departamento de Microbiologia e Parasitologia, Universidade Federal de Santa Catarina, Caixa Postal 476, 48040-900 Florianopolis, SC, Brasil ${ }^{+}$Corresponding author. Fax: +970-491-0603. E-mail: rtitus@vines.colostate.edu. Received 20 July 1998 Accepted 3 November 1998
}

profile (IFN-g ${ }^{\text {lo; }}$ IL-4 $\left.{ }^{\text {hi }}\right)$. IL-4 can block the ability of IFN-g to activate MØs to kill Leishmania (Lehn et al. 1989, Liew et al. 1989).

In contrast to infection with $L$. major, $L$. braziliensis induces only a transient cutaneous disease, even in BALB/c mice. This may at least in part be the explanation for why little experimental work has been performed with L. braziliensis (Neal $\&$ Hale 1983, Childs et al. 1984). We recently reported (DeKrey et al. 1998) that following infection with $L$. braziliensis or L. major, BALB/c mice produced similar levels of IFN-g. However, $L$. braziliensis infected mice produced much less IL-4 (approximately 10-fold). In addition, when the L. braziliensis infected mice were treated with a neutralizing anti-IFN-g, the animals were unable to resolve their infection. We concluded that BALB/c mice cure an infection with L. braziliensis because the low levels of IL-4 they produce are unable to block the ability of IFN-g to activate $L$. braziliensis infected MØs to kill the parasite.

Resolution of an infection with a particular species of Leishmania usually confers complete resistance to re-challenge with the same parasite. However, in addition to this, a primary infection with a given species of Leishmania can also confer cross-protection against a different species of Leishmania (Lainson \& Bray 1966, Lainson \& Shaw 1977, Alexander \& Phillips 1978a,b, Perez et al. 1979, Alexander 1988, Neal et al. 1990, Peters et al. 1990, Melby 1991, Abramson et al. 1995, Gicheru et al. 1997). Cross-protection has been 
shown in several different mammalian hosts; the protection sometimes acts in only one direction (Lainson \& Shaw 1977), and in some cases the sex of the host influences the cross-protection seen (Alexander 1988).

Since $L$. braziliensis is unable to trigger a strong $\mathrm{Th} 2$ response in BALB/c mice, we hypothesized that following resolution of an infection with $L$. braziliensis, $\mathrm{BALB} / \mathrm{c}$ mice might be at least partially protected against challenge with $L$. major. We report here that previous exposure to $L$. braziliensis can confer complete protection against a subsequent challenge with $L$. major and that this protection is dependent upon IFN-g production.

\section{MATERIALS AND METHODS}

Mice and parasites - Young adult female mice were used in all experiments. BALB/c mice were obtained from either the National Cancer Institute (Bethesda, MD) or Jackson Laboratory (Bar Harbor, ME). C57BL/6 were obtained from the National Cancer Institute. Stationary phase promastigotes of L. braziliensis (MHOM-BR-79LTB111) or L. major (RHO-SU-59-P) were used. Parasites were maintained as described (Titus et al. 1984).

Infecting mice and determining parasite numbers in cutaneous lesions - Mice were injected with the numbers of promastigotes indicated in the text in one hind footpad and lesion development was followed by measuring the thickness of the infected footpad compared to the thickness of the same footpad prior to infection.

Parasite numbers were determined in infected footpads using a published limiting dilution assay for determining parasite burdens in infected mouse tissues (Lima et al. 1997).

In some experiments mice were treated with a neutralizing anti-IFN-g(XMG1.2) antibody as described in DeKrey et al. (1998).

Determining levels of cytokines in culture supernatants - At various times after infection, 3-5 mice per group were killed for evaluation. Single cell suspensions were prepared from draining lymph nodes (inguinal and popliteal). Cells were adjusted to $5 \times 10^{6} / \mathrm{ml}$ in Dulbecco's modified Eagle medium (Maryanski et al. 1982) containing 0.5\% normal mouse serum (Harlan Bioproducts, Indianapolis, IN). Cultures were stimulated with $10^{6} \mathrm{~L}$. major promastigotes $/ \mathrm{ml}$ and the supernatant of the cultures was harvested $72 \mathrm{hr}$ later (a time determined to be optimal for the cytokines examined) for analysis.

Levels of IFN-g and IL-4 in culture supernatants were determined by enzyme-linked immunosorbent assay (ELISA) using techniques published elsewhere (Soares et al. 1997).
Statistical analysis - Significance was determined using an non-paired $t$ test. Differences were considered to be significant when $\mathrm{p}<0.05$.

All experiments shown are representative of two to three independent experiments.

\section{RESULTS}

To determine whether previous exposure to $L$. braziliensis led to protection against a subsequent challenge with $L$. major, we first experimented with the dose of $L$. braziliensis and the time between infection with $L$. braziliensis and challenge with $L$. major. We found that a large dose of $L$. braziliensis $\left(10^{7}\right)$ administered subcutaneously (s.c.) in one hind footpad led to complete protection against a subsequent challenge with $10^{6} \mathrm{~L}$. major s.c. in the opposing hind footpad (Fig. 1). Moreover, the protective effect of pre-infecting with $L$. braziliensis was a dose titratable phenomenon. As shown in Fig. 1, a dose of $10^{3} \mathrm{~L}$. braziliensis led to the least protection against challenge with $L$. major whereas a dose of $10^{7} \mathrm{~L}$. braziliensis led to the greatest protection. Lesions of $L$. major were the largest in mice pre-treated with $10^{3}$ L. braziliensis and only $20 \%$ of the mice (see numbers in the legend of Fig. 1) cured these $L$. major-induced lesions; in contrast, lesions of $L$. major were the smallest in mice pre-treated with $10^{7}$ L. braziliensis and $100 \%$ of the mice cured these L. major-induced lesions.

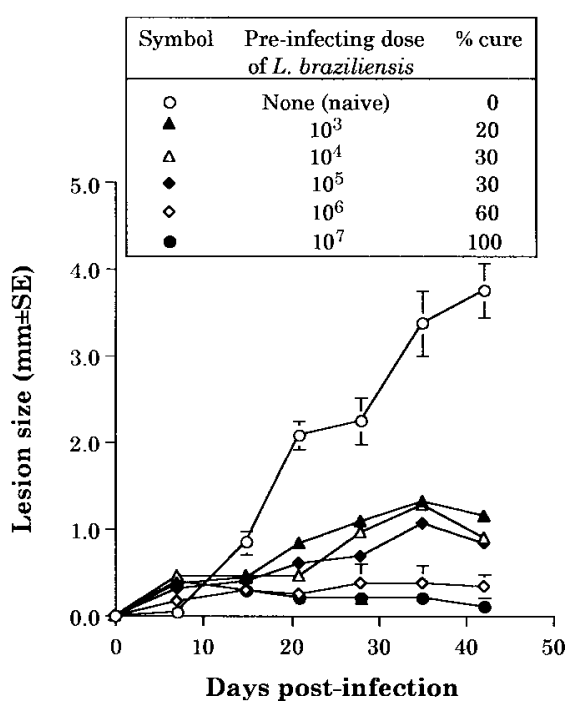

Fig. 1: course of infection with Leishmania major in BALB/c mice pre-infected with different concentrations of $L$. braziliensis. Groups of $10 \mathrm{BALB} / \mathrm{c}$ mice each were pre-infected with the indicated doses of $L$. braziliensis s.c. in one hind footpad. Twelve weeks later these animals were challenged s.c. in the opposing hind footpad with $10^{6} \mathrm{~L}$. major. Controls consisted of naive mice infected with $10^{6} \mathrm{~L}$. major. Lesions were monitored as described in Materials and Methods. 
We also determined that the degree of resistance to challenge with $L$. major increased with time after exposure to $L$. braziliensis. Little if any protection against challenge with $L$. major was achieved when the two parasites were injected simultaneously. Some protection was observed when mice were challenged with L. major at 6 or 8 weeks after exposure to L. braziliensis. However, $100 \%$ protection against challenge with $L$. major was consistently achieved only at 12 weeks after exposure to L. braziliensis (data not shown). Importantly, at 12 weeks post-L. braziliensis injection, we were also unable to detect viable $L$. braziliensis in treated mice by limiting dilution analysis (data not shown). Therefore, for the remaining experiments presented here, mice were treated with $10^{7}$ L. braziliensis and challenged 12 weeks later with $10^{6}$ L. major.

The experiment shown in Fig. 1 demonstrated that pre-infection with L. braziliensis allows $\mathrm{BALB} / \mathrm{c}$ mice to control the outgrowth of lesions of $L$. major when the mice were challenged with the parasite. To determine whether this was accompanied by destruction of $L$. major in the lesions, we measured the parasite burdens in the lesions. In L. braziliensis-naive control mice, $L$. major continued to replicate through day 42 of infection (Table I). In contrast, in mice pre-infected with $L$. braziliensis 12 weeks earlier, L. major was destroyed such that by day 42 of the experiment there were approximately 2,000-fold fewer parasites in their lesions compared to control mice (Table I).

We next analyzed the mechanism underlying the protection seen against challenge with $L$. $m a$ jor in BALB/c mice pre-infected with $L$. braziliensis. We first noted that an intense swelling response occurred in the footpads of $L$. braziliensis pre-treated mice when the mice were challenged with L. major (Fig. 2). This swelling response was characteristic of delayed-type hypersensitivity (DTH) in that it peaked from 24 to 48 hr post-challenge with $L$. major and it persisted to $72 \mathrm{hr}$ post-challenge (Fig. 2). This observation suggested that cross reactive antigenic epitopes exist in L. braziliensis and L. major that prime $\mathrm{T}$ cell responses. Moreover, since DTH is mediated by Th1-type T cells (Mosmann \& Coffman 1989), this also suggested that infection with $L$. braziliensis triggered Th1 T cells in BALB/c mice that could recognize L. major antigen(s) when the mice were challenged with the parasite.

To test the hypothesis that cross reactive Th1 T cells were elicited by pre-infection with $L$. braziliensis, we measured the cytokines produced when lymph node cells from $L$. braziliensis preinfected mice were challenged with $L$. major in vitro. We first harvested the popliteal and inguinal nodes draining the footpad of mice pre-infected with $L$. braziliensis 12 weeks earlier. These cells were stimulated with $L$. major promastigotes in

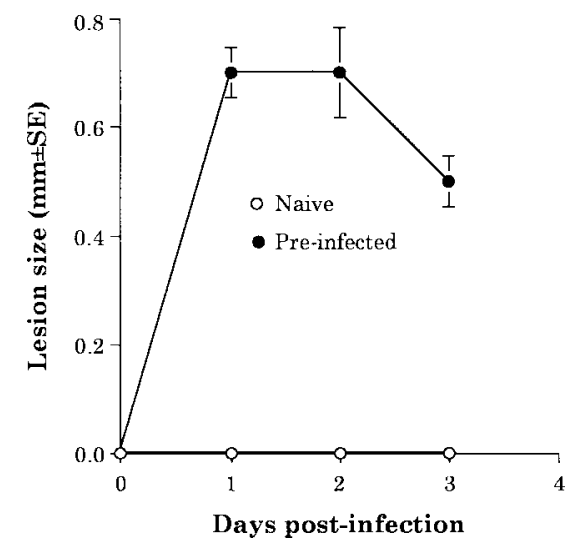

Fig. 2: footpad swelling response of Leishmania braziliensis pre-infected BALB/c mice challenged with L. major. BALB/c mice were pre-infected with $L$. braziliensis and challenged with L. major as described in the legend of Fig. 1.

TABLE I

Numbers of Leishmania major in lesions of BALB/c mice pre-infected with L. braziliensis

\begin{tabular}{lcc}
\hline $\begin{array}{l}\text { Days post- } \\
\begin{array}{l}\text { L. major } \\
\text { infection }\end{array}\end{array}$ & Number of $L$. major/footpad lesion $(95 \%$ confidence limits) \\
\cline { 2 - 3 } & Naive & Pre-infected \\
\hline 3 & $0.24 \times 10^{5}(0.06-0.43)$ & $0.04 \times 10^{5}(0.01-0.07)^{a}$ \\
7 & $2.85 \times 10^{5}(1.06-4.63)$ & $0.40 \times 10^{5}(0.10-0.71)$ \\
21 & $35.77 \times 10^{5}(9.63-61.90)$ & $3.75 \times 10^{5}(1.40-6.05)$ \\
42 & $79.75 \times 10^{5}(23.70-135.80)$ & $0.40 \times 10^{5}(0.01-0.08)$ \\
\hline
\end{tabular}

a: BALB/c mice were infected with $10^{7}$ L. braziliensis s.c. in a hind footpad. Twelve weeks later, the mice were challenged in the opposing footpad with $10^{6}$ L. major. Controls consisted of age-matched L. braziliensis-naive $\mathrm{BALB} / \mathrm{c}$ mice challenged with $10^{6} \mathrm{~L}$. major. At the indicated time points after challenge, the footpad lesions from duplicate mice of each group were subjected to limiting dilution analysis to determine the numbers of $L$. major present. 
vitro and the supernatants were harvested $72 \mathrm{hr}$ later to determine their content of IFN-gand IL-4. These lymph node cells produced substantial amounts of IFN-g $(15.48 \mathrm{ng} / \mathrm{ml}$, Table II) but no detectable IL4. Moreover, when the same lymph node cells were harvested from L. braziliensis pre-infected mice at varying times after the mice were challenged with L. major, the cells continued to produce substantially more IFN-g but less IL-4 than control naive $\mathrm{BALB} / \mathrm{c}$ mice challenged with L. major (Table II).

Since L. braziliensis pre-infected mice produced elevated levels of IFN-g but lower levels of IL-4 compared to naive mice when the mice were challenged with $L$. major (Table II), we next tested the hypothesis that the IFN-g was involved in the protection seen against challenge with $L$. major. $L$. braziliensis pre-infected $\mathrm{BALB} / \mathrm{c}$ mice were treated with a neutralizing IFN-g antibody as described in the Materials and Methods and challenged with $L$. major. As can be seen in Fig. 3, treating with anti-

\section{TABLE II}

Cytokines produced by lymph node cells from Leishmania braziliensis pre-infected BALB/c mice following challenge in vitro with L. major

\begin{tabular}{lcc}
\hline $\begin{array}{l}\text { Days post- } \\
\text { L. major } \\
\text { infection }\end{array}$ & \multicolumn{2}{c}{ Levels of cytokine produced by } \\
\cline { 2 - 3 } IFN-g $^{a}(\mathrm{ng} / \mathrm{ml} \pm \mathrm{SD})$ & Naive & Pre-infected \\
$0^{*}$ & & \\
3 & None detected & $15.48 \pm 0.47 \mathrm{c}$ \\
$7 *$ & $23.12 \pm 6.26$ & $16.38 \pm 2.14$ \\
$21^{*}$ & $16.39 \pm 9.64$ & $80.30 \pm 7.47$ \\
42 & $23.23 \pm 1.95$ & $45.78 \pm 3.02$ \\
$\mathrm{IL}^{b}(\mathrm{pg} / \mathrm{ml} \pm \mathrm{SD})$ & $8.05 \pm 5.24$ & $14.09 \pm 0.54$ \\
0 & & \\
3 & None detected & None detected \\
7 & None detected & $60.74 \pm 25.77$ \\
$21^{*}$ & $751.63 \pm 250.59$ & $280.95 \pm 61.73$ \\
$42^{*}$ & $326.57 \pm 27.93$ & $98.24 \pm 38.27$ \\
\hline
\end{tabular}

$a$ : interferon-g; $b$ : interleukin-4; $c$ : groups of BALB/c mice were infected with $10^{7} \mathrm{~L}$. braziliensis s.c. in a hind footpad. Twelve weeks later, the lesion-draining popliteal and inguinal lymph node cells from some of the mice were harvested and restimulated with $L$. major promastigotes as described in Materials and Methods. The remaining mice were challenged in the opposing footpad with $10^{6} \mathrm{~L}$. major. Controls consisted of agematched L. brasiliensis-naive BALB/c mice challenged with $10^{6} \mathrm{~L}$. major. At the indicated time points after challenge, cells from lymph nodes draining the L. majorchalleged footpad were stimulated with L. major in vitro. Seventy-two hr later, the levels of IFN-gand IL-4 present in the supernatants of the cultures were determined by ELISA as described in Materials and Methods. The asterisks in the table indicate a statistically significant difference $(\mathrm{P}<0.05)$ between groups.
IFN-gabrogated the protection seen when the mice were challenged with $L$. major.

Finally, since L. braziliensis pre-infected $\mathrm{BALB} / \mathrm{c}$ mice were protected against challenge with $L$. major, we tested whether resistance to infection with $L$. major was enhanced in a mouse that normally cures an infection with the parasite. C57BL/6 mice were pre-infected with $10^{7} \mathrm{~L}$. braziliensis and 12 weeks later were challenged with $10^{6}$ L. major. As can be seen in Fig. 4, these mice showed markedly increased resistance to challenge with L. major.

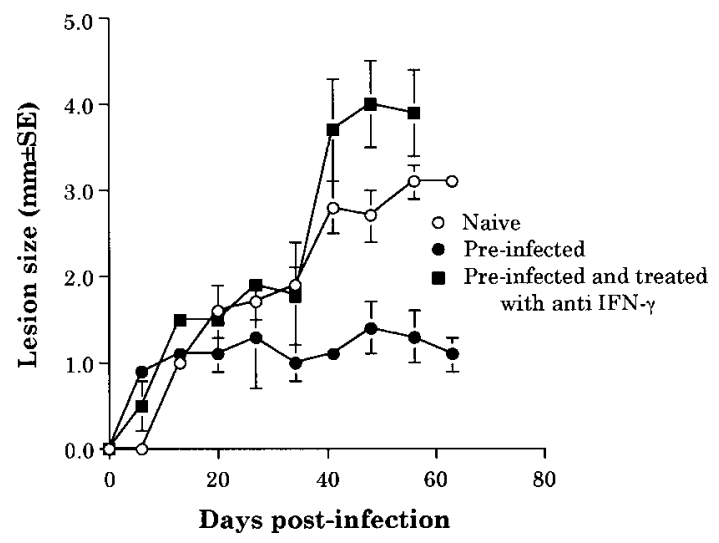

Fig. 3: Leishmania braziliensis pre-infected BALB/c mice do not resist infection with $L$. major when the animals are treated with anti-IFN-gantibody. Groups of BALB/c mice were pretreated with $10^{7} \mathrm{~L}$. braziliensis and 12 weeks later the mice were challenged with $10^{6} \mathrm{~L}$. major. One of the groups of pretreated mice was also injected with a neutralizing anti-IFN-g antibody as described in Materials and Methods. Controls consisted of age-matched $L$. braziliensis-naive BALB/c mice challenged with $L$. major. Lesions were monitored as described in Materials and Methods.

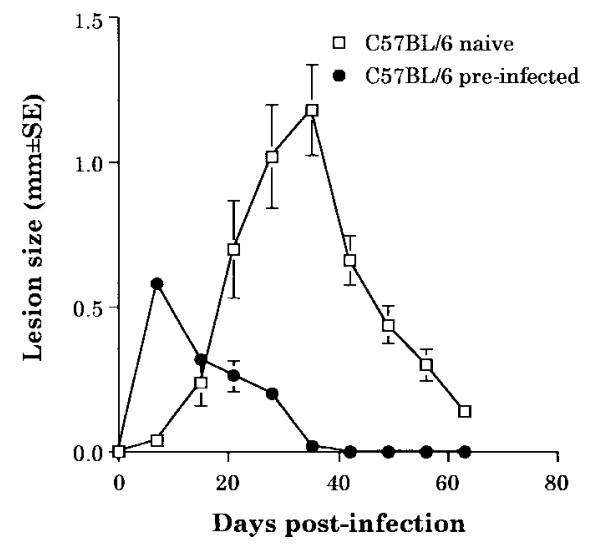

Fig. 4: pre-infecting with Leishmania braziliensis enhances the resistance of C57BL/6 mice to challenge with $L$. major. C57BL/6 mice were pre-infected with $10^{7} \mathrm{~L}$. braziliensis promastigotes and 12 weeks later the mice were challenged with $10^{6}$ L. major. Controls were L. braziliensis-naive C57BL/6 mice challenged with L. major. Lesions were monitored as described in Materials and Methods. 


\section{DISCUSSION}

We recently reported that cutaneous lesions develop on $\mathrm{BALB} / \mathrm{c}$ mice following infection with either L. major or L. braziliensis; however, while BALB/c mice ultimately die of infection with $L$. major, the mice resist infection with L. braziliensis, kill the parasite, and heal their cutaneous lesions (DeKrey et al. 1998). Further analysis of this system revealed that following infection with $L$. $m a$ jor, BALB/c mice developed a Th2-biased immune response. In contrast, following infection with $L$. braziliensis, the mice developed a Th1-biased response (DeKrey et al. 1998). Since a Th1 response mediates cure in mice infected with L. major, we hypothesized that BALB/c mice that had cured an infection with $L$. braziliensis would resist challenge with $L$. major. To our knowledge, no one has examined whether pre-infecting mice with $L$. braziliensis confers protection against challenge with L. major.

We report here that pre-infecting BALB/c mice with $L$. braziliensis confers full protection against challenge with $L$. major. That is, whereas $L$. braziliensis-naive control mice were susceptible to infection with L. major, L. braziliensis pre-treated mice resolved cutaneous lesions of $L$. major (Fig. 1 ) and destroyed L. major parasites within the lesions (Table I).

Full protection against $L$. major required preinfection with a high dose of L. braziliensis $\left(10^{7}\right.$, Fig. 1). The reason such a high dose of $L$. braziliensis was required is not known. However, L. braziliensis is known to be poorly infective for laboratory mice (Samuelson et al. 1991). Since the metacyclic form of Leishmania is the infective form of the parasite [the form that survives and efficiently infects MØs in the vertebrate host (da Silva et al. 1989, Puentes et al. 1990)], it is possible that conversion to metacyclics by $L$. braziliensis is very inefficient using standard culture conditions. Thus, a large dose of L. braziliensis is required to successfully infect antigen presenting cells such as MØs in the host which in turn stimulate an effective immune response.

In addition to requiring a large dose of $L$. braziliensis to achieve full protection against challenge with $L$. major, it was also necessary to wait 12 weeks before challenging with the parasite, a time when L. braziliensis parasites could not be detected in the mice. Indeed, little if any protection was achieved when L. braziliensis and L. major parasites were injected simultaneously. Taking these observations together, in conjunction with the fact that $L$. braziliensis pre-treated mice produced large amounts of IFN-g when challenged with L. major (Table II), suggests that protection against challenge with $L$. major was not mediated by either concomitant immunity or a non-specific inflammatory response against $L$. major. Rather, the data suggest that there are antigenic epitopes shared between L. braziliensis and L. major. When a BALB/c mouse is infected with $L$. braziliensis, these epitopes elicit a protective $\mathrm{Th} 1 \mathrm{~T}$ cell response such that the mice mount a Th1 response when challenged with L. major. The nature of the crossreactive epitopes of $L$. braziliensis and $L$. major are unknown but are currently under investigation. It would be interesting if these cross-reactive epitopes were found to be expressed at low levels on $L$. braziliensis and/or to stimulate rare $\mathrm{T}$ cell clones. If this were the case, it would offer an alternative explanation for our observations that a large dose of $L$. braziliensis was required to achieve full-protection against $L$. major challenge and that it required 12 weeks for this protection to develop.

In conclusion, the data presented here confirm and further characterize previous reports that demonstrated cross-protection between different species of Leishmania. In addition, the data show that BALB/c mice can be induced to mount a protective Th1 response against a normally lethal infection with $L$. major, and that this can occur in the absence of intervention with cytokines or anticytokines.

\section{ACKNOWLEDGMENTS}

To Monica Estay for excellent technical assistance.

\section{REFERENCES}

Abramson MA, Dietze R, Frucht DM, Schwantz R, Kenney RT 1995. Comparison of New and Old World leishmaniasis in an endemic region of Brazil. Clin Infect Dis 20: 1292-1297.

Alexander J 1988. Sex differences and cross-immunity in DBA/2 mice infected with $L$. mexicana and $L$. major. Parasitology 96: 297-302.

Alexander J, Phillips RS 1978a. Leishmania mexicana and $L$. tropica: inhibition of growth in mice by concurrent infections of Trypanosoma brucei. Exp Parasitol 44: 136-142.

Alexander J, Phillips RS 1978b. Leishmania tropica and Leishmania mexicana: cross-immunity in mice. Exp Parasitol 45: 93-100.

Bogdan C, Gessner A, Rollinghoff M 1993. Cytokines in leishmaniasis: a complex network of stimulatory and inhibitory interactions. Immunobiology 189 : 356-396.

Childs GE, Lighther LK, McKinney LA, Groves M, Price E, Hendricks L 1984. Inbred mice as model hosts for cutaneous leishmaniasis. I. Resistance and susceptibility to infection with Leishmania braziliensis, L. mexicana and L. aethiopica. Ann Trop Med Parasitol 78: 25-34.

da Silva RP, Hall BF, Joiner KA, Sacks DL 1989. CR1, the $\mathrm{C} 3 \mathrm{~b}$ receptor, mediates binding of infective Leishmania major metacyclic promastigotes to human macrophages. J Immunol 143: 617-622. 
DeKrey GK, Lima HC, Titus RG 1998. Analysis of the immune responses of mice to infection with Leishmania braziliensis. Infect Immun 66: 827-829.

Gicheru MM, Olobo JO, Anjili CO 1997. Heterologous protection by Leishmania donovani for Leishmania major infections in the vervet monkey model of the disease. Exp Parasitol 85: 109-116.

Lainson R, Bray RS 1966. Studies on the immunology and serology of leishmaniasis. II. Cross-immunity experiments among different forms of American cutaneous leishmaniasis in monkeys. Trans $R$ Soc Trop Med Hyg 60: 526-532.

Lainson R, Shaw JJ 1977. Leishmaniasis in Brazil: XII. Observations on cross-immunity in monkeys and man infected with Leishmania mexicana mexicana, L. m. amazonensis, L. braziliensis braziliensis, L. b. guyanensis and L. b. panamensis. J Trop Med Hyg 80: 29-35.

Lehn M, Weiser WY, Engelhorn S, Gillis S, Remold HG 1989. IL-4 inhibits $\mathrm{H}_{2} \mathrm{O}_{2}$ production and antileishmanial capacity of human cultured monocytes mediated by IFN-g. J Immunol 143: 3020-3024.

Liew FY, O’Donnell CA 1993. Immunology of leishmaniasis. Adv Parasitol 32: 161-259.

Liew FY, Millott S, Li Y, Lelchuk R, Chan WL, Ziltener H 1989. Macrophage activation by interferon-gfrom host-protective T cells is inhibited by interleukin (IL) 3 and IL-4 produced by disease-promoting T cells in leishmaniasis. Eur J Immunol 19: 1227-1232.

Lima HC, Bleyenberg J, Titus RG 1997. A simple method for quantifying Leishmania in tissues of infected animals. Parasitol Today 13: 80-82.

Maryanski J, van Snick J, Cerottini J-C, Boon T 1982. Immunogenic variants obtained by mutagenesis of mouse mastocytoma P 815. III. Clonal analysis of the syngeneic cytolytic T lymphocyte response. Eur J Immunol 12: 401-405.

Melby PC 1991. Experimental leishmaniasis in humans: review. Rev Infect Dis 13: 1009-1017.

Mosmann TR, Coffman RL 1989. Th1 and Th2 cells: different patterns of lymphokine secretion lead to different functional properties. Ann Rev Immunol 7: 145-173.

Murray HW, Rubin BY, Rothermel CD 1983. Killing of intracellular Leishmania donovani by lymphokine-stimulated human monocytes. Evidence that interferon $\mathrm{g}$ is the activating lymphokine. J Clin Invest 72: 1506-1510.
Nacy CA, Fortier AH, Meltzer MS, Buchmeier NA, Gray PW 1985. Macrophage activation to kill Leishmania major. Activation of macrophages for intracellular destruction of amastigotes can be induced by both recombinant interferon-g and non-interferon lymphokines. J Immunol 135: 3505-3511.

Neal RA, Hale C 1983. A comparative study of susceptibility of inbred and outbred mouse strains compared with hamsters to infection with New World cutaneous leishmaniases. Parasitology 87: 7-13.

Neal RA, Reeves A, Peters W 1990. Leishmania infecting man and wild animals in Saudi Arabia. 7. Partial protection of mice against Leishmania major by prior infection with L. arabica. Trans $R$ Soc Trop Med Hyg 84: 233-238.

Perez H, Arredondo B, Machado R 1979. Leishmania mexicana and Leishmania tropica: cross immunity in C57BL/6 mice. Exp Parasitol 48: 9-14.

Peters W, Bryceson A, Evans DA, Neal RA, Kaye P, Blackwell J, Killick-Kendrick R, Liew FY 1990. Leishmania infecting man and wild animals in Saudi Arabia. 8. The influence of prior infection with Leishmania arabica on challenge with $L$. major in man. Trans $R$ Soc Trop Med Hyg 84: 681-689.

Puentes SM, da Silva RP, Sacks DL, Hammer CH, Joiner K 1990. Serum resistance of metacyclic stage Leishmania major promastigotes is due to release of C5b9. J Immunol 145: 4311-4316.

Reed SG, Scott P 1993. T-cell and cytokine responses in leishmaniasis. Curr Opin Immunol 5: 524-531.

Reiner SL, Locksley RM 1995. The regulation of immunity to Leishmania major. Ann Rev Immunol 13: 151-177.

Samuelson J, Lerner E, Tesh R, Titus R 1991. A mouse model of Leishmania braziliensis braziliensis infection produced by co-injection with sand fly saliva. J Exp Med 173: 49-54.

Soares MBP, David JR, Titus RG 1997. An in vitro model for infection with Leishmania major that mimics the immune response in mice. Infect Immun 65: 2837-2845.

Titus RG, Kelso A, Louis JA 1984. Intracellular destruction of Leishmania tropica by macrophages activated with macrophage activating factor/interferon. Clin Exp Immunol 55: 157-165.

Titus RG, Theodos CM, Shankar A, Hall LR 1994. Interactions between Leishmania major and macrophages. Immunol Series 60: 437-459. 